\title{
An innovative survey of urban systems dynamics: the evidence of the MoTo project
}

\author{
N. Marchettini ${ }^{1}$, R. M. Pulselli ${ }^{1} \&$ E. B. P. Tiezzi ${ }^{2}$ \\ ${ }^{I}$ Ecodynamics Group, Department of Chemistry, \\ University of Siena, Italy \\ ${ }^{2}$ Department of Mathematics and Informatics, University of Siena, Italy
}

\begin{abstract}
Is our ability to study urban systems and their sustainability exhaustive enough? Are our tools capable of understanding the complexity of contemporary cities? Nowadays, it is necessary to develop new techniques for investigating dynamic processes, within urban systems, that are not directly observable. We ask "how", but, most of all, "when", "how long" and "how intensely" city users move and live within urban space.

In recent years, new monitoring techniques have been developed in order to collect information on urban dynamics and provide tools for managing services and infrastructures in urban systems that seem to escape control. The challenge is to improve our understanding of regions and translate this knowledge into good practices of planning and development.

In particular, the research presented in this paper is based on a new method for monitoring urban dynamics, which elaborates data from cell phones through GIS tools, to provide a sort of "dynamic census" of population. This was expected to measure, even if approximately, the intensity of use of urban spaces by citizens.

Results from the "MoTo" project (Mobile Toscana) - a study of the metropolitan area of central Tuscany, including Florence (Italy) - were presented. Outcomes demonstrated a high relevance of cell phone activity data and a promising efficiency of the technique presented in elaborating information through GIS tools for monitoring human activities in a wide metropolitan area. Keywords: monitoring technique, mobile telecommunication technology, geographic information systems, geo-demographic system, urban studies.
\end{abstract}




\section{Introduction}

Today we are witnessing the continued growth of urban systems where more than $50 \%$ of the world's population lives [1]. This phenomenon leads to problems of congestion, mobility, air pollution, resource overuse, waste management and loss of ecosystems (e.g. lack of parks and natural areas).

This is also evident at the local scale. As a case study, we investigated the metropolitan area of central Tuscany (Italy), around Florence, since this is officially considered one of nine Italian metropolitan areas together with Milan, Bologna, Genoa, Bari, Naples, Turin, Venice and Rome. This includes the main cities of Florence, Prato, Pistoia and Empoli and covers about $1575 \mathrm{~km}^{2}$ with a population of $1,200,000$ inhabitants.

In managing this area, the administration has to face different problems. Some of these are: a) congestion, air pollution and urban decay within and around the city of Florence as an important centre of cultural heritage; b) physical growth of urban systems, mainly occurring spontaneously and not systematically in the last fifty years; c) increasing burden of population, buildings, and activities with environmental impacts that is still supported by urban infrastructures built in the late nineteenth century and never improved or readapted. Faced with this scenario, it is an urgent priority to study the functioning of the urban system as a whole and to develop new investigating tools.

This was the aim of the project MoTo (Mobile Tuscany), funded by the regional administration of Tuscany, that developed a population density monitoring technique, tested for the first time at the Massachusetts Institute of Technology in 2004 and further elaborated by the researchers of Siena [2-6]. This allowed for observing the movements and dynamics of city users (those carrying a mobile device) in relation to the physical space of the city. In particular, thanks to the diffused use of mobile technologies and their capillary distribution throughout the territory, it is possible to monitor, with a good approximation, the activities of a community in a specific region and their evolution over time. Mobile telecommunications were used therefore as tools for retrieving statistical data that is reliable and detailed (data is collected in an aggregated and anonymous form); Geographic Information Systems were used to elaborate data and provide innovative solutions for visualizing phenomena that are variable in time.

This is expected to identify nodes, preferential pathways and urban attractors, and support the territorial planning practices by revealing time and intensity of use of urban space.

\section{Method: monitoring data from telecommunication technologies}

On the basis of data regarding the diffusion of mobile phones in Italy, it is possible to assume that the variation in intensity of use corresponds proportionately to a variation in population density. This consideration has been 
confirmed by recent statistics on mobile telecommunications in Europe and in Italy that indicate a high percentage of stable users of mobile phones (Source: EITO 2007 - European Information Technology Observatory).

The capillary diffusion of mobile telecommunication technologies is based on an articulated system of radio transmission, based on a dense network of radio base stations and antennas. Technically, every radio base station is the physical support of many antennas; each antenna transmits data in a direction on a set of radio channels different from those utilized by the adjacent antennas, in order to avoid interferences. Thus, the field of action of a single antenna can be identified as a confined area, namely cell.

In this research, compared to the previous studies, the cases of Milan [2-4] and Pescara [5, 6], data was collected in a more accurate way: cells were $550 \mathrm{~m}$ diameter and the monitored activity was found to be mainly concentrated $(90 \%)$ within a ray of $4400 \mathrm{~m}$ from the antenna. A set of 50944 cells where analyzed in the region under study. Moreover, it was evident that the telecommunication network is tighter within high populated urban areas and sparser in the rural areas. In order to handle this amount of data, the irregular cell system was transferred into a geometric grid consisting of $500 \times 500 \mathrm{~m}$ pixels. Data, a value that corresponds to the number of calls made within each cell, was thus associated to each pixel in the grid (which may correspond to more cells).

Variations in intensity were represented through sequences of maps (the different values of traffic intensity correspond proportionately to levels of a chromatic scale) and also aggregated for comparing different hours, days (i.e. working days, Saturdays, Sundays or holydays) or seasons. This made possible to visualize time and intensity of use of urban space and to analyze the general functioning of the whole territorial system.

\section{The metropolitan area of central Tuscany: results and discussion}

The metropolitan area of central Tuscany includes areas with different properties, functions, and problems. By virtue of this richness and variety, it represents a symbolic case study. The region under study is marked by the presence of a long valley crossed by the river Arno. The city of Florence, in the east side, is within a ring of small and medium centres, such as Sesto Fiorentino, Fiesole, Bagno a Ripoli, Galluzzo, and Scandicci. The western side includes the cities of Prato, Pistoia and Empoli.

Trends of cell phone activity in 24-hours show that the activity is mainly concentrated in the daytime hours and drastically reduced during the night; that is, the activity "turns on" early in the morning and "turns off" almost completely in the night. Furthermore, we found that the intensity of activity is reduced during Sundays with respect to the other days of the week and, especially on Saturday evenings, it persists until the late night. In all these cases, it is curious to notice a decrease of calls around 3 p.m., indicating a habit or a custom of local population. These first analyses thus highlighted a repetitive phenomenon that characterizes and is representative of human behaviour in the region. 
The application of GIS tools made possible to visualize spatial patterns of intensity of activity, representing indirectly the presence of people. This description of urban dynamics and their evolution in space and time allowed for an analysis both qualitative and quantitative or the geo-demographic system.

A schematic classification of homogenous areas within the region was provided in order to synthetically express the results obtained through dynamic maps. Based on intensity of activity, three classes of space use (high, medium, and low) were identified (Figure 1).

Areas in the highest class include urban spaces with a high level of urbanization, in which places of work, services, and residences are mainly located; areas with a low intensity are predominantly rural; the zones with medium intensity are in-between areas among the main urban centres. The latter is a result of urban sprawl and an evidence of the spontaneous formation of a diffused and continuous urban system which tended to delocalize functions along the connecting arteries. The configuration represented in Figure 1 appears stable in different days and seasons.

Based on the distribution of the activity in different areas, known areas with given functions and settlements were identified and compared to each other through a diagram (Figure 2). About 45\% of the overall activity was concentrated around the three main cities of Florence, Prato and Pistoia with different intensities.

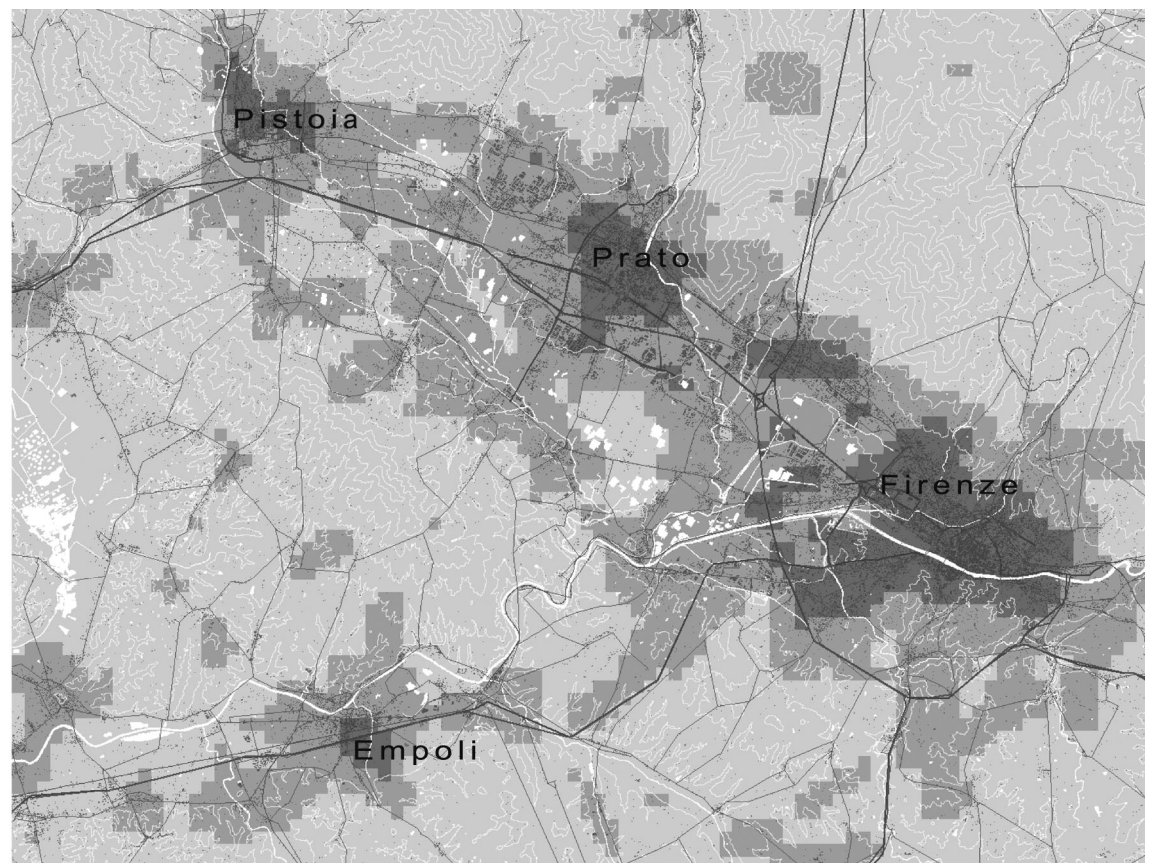

Figure 1: Geo-demographic organization of the metropolitan area of central Tuscany. Areas with high, medium and low intensity of activity on a working spring day. 


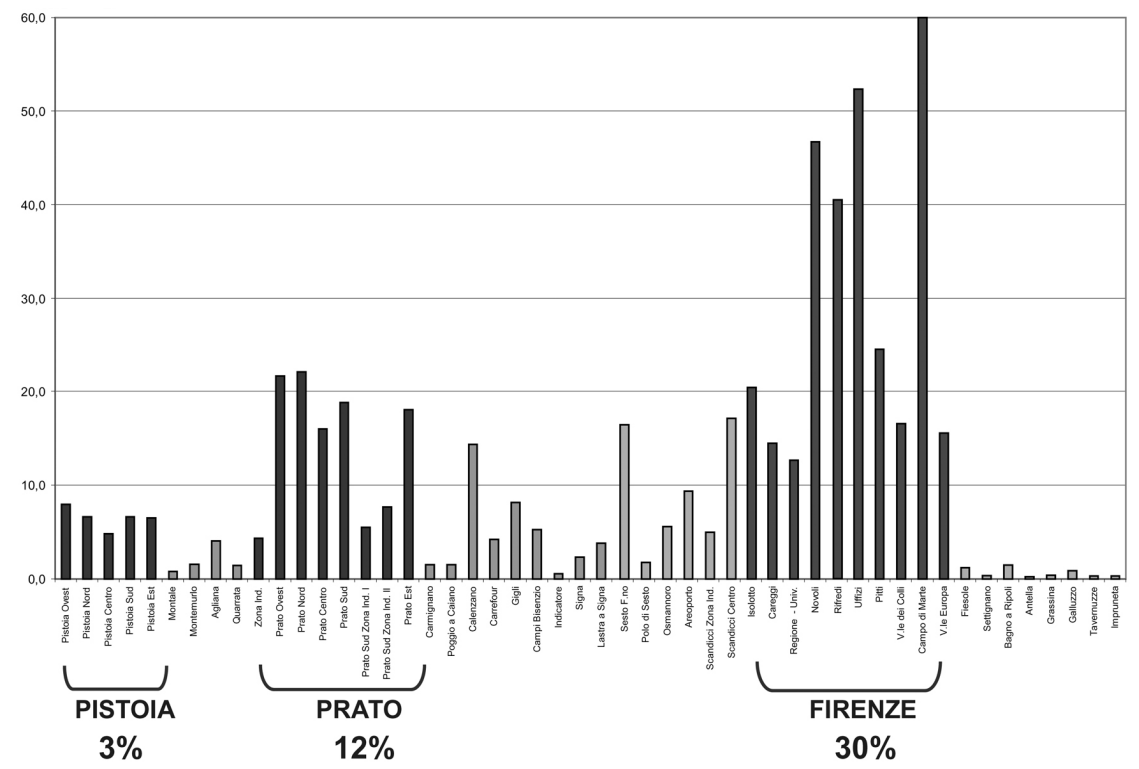

Figure 2: Diagram of intensity of activity in different places along the axis Pistoia - Florence.

\section{Conclusion}

This paper presented results from a monitoring technique based on mobile telecommunication technologies. Data of cell phones activity was used for representing urban dynamics in real time. Chromatic patterns plotted on sequences of maps showed the variation of intensity of cell phones activity that corresponds proportionally to population density. Outcomes allowed for the understanding of the geo-demographic system and its functioning within the metropolitan area of central Tuscany. Aggregated data, with reference to particular locations and urban functions, were also analyzed and compared for measuring variation in urban space use intensity. The MoTo project therefore provided information for a qualitative and quantitative analysis of the relations between the parts (e.g. main centres and suburbs) of the urban system, conceived as a whole highly integrated system.

\section{Acknowledgements}

The MoTo project was selected from the competition for research and innovation in the territorial and environmental field, and funded by the General Directorate of Territorial and Environmental Policies of the Tuscan Region. We thank the team of the mobile telecommunication company Telecom Italia, particularly Dr. Daniele Nutini, for collaborating with us and making this project possible. 


\section{References}

[1] United Nations. World Urbanization Prospects: The 2005 Revision. United Nations, New York 2006.

[2] C. Ratti, R.M. Pulselli, S. Williams, D. Frenchman. Mobile Landscapes: using location data from cell phones for urban analysis. Environment and Planning B: Planning and Design 33(5) 2006 727-748.

[3] Pulselli R M, Ratti C, Tiezzi E. City out of chaos: social patterns and organization in urban systems. International Journal of Ecodynamics 1(2) 2006 125-134.

[4] Pulselli R M, Romano P, Ratti C, Tiezzi E. Computing urban mobile landscapes through monitoring population density based on cell-phone chatting. International Journal of Design \& Nature and Ecodynamics 3 (2) 2008 121-134.

[5] Bastianoni S, Pulselli R M, Romano P, Pulselli F M. Dynamics and evolution of urban patterns: the evidence of the Mobile Landscapes project. (Ed.) Brebbia C A. Design \& Nature IV-Comparing Design in Nature with Science and Engineering. WIT Press. Southampton, UK 2008 253-260.

[6] Pulselli R M, Romano P. Dinamiche dei sistemi urbani. Indagine di un'area metropolitana; Urban systems dynamics. The investigation of a metropolitan area. Alinea, Florence 2009. 